\title{
Three-Body System with Short-Range Interactions
}

\author{
J. Gegelia \\ Department of Physics, The Flinders University of South Australia, \\ Bedford Park, SA 5042, Australia
}

(November 28, 2018)

\begin{abstract}
Within the framework of non-relativistic scalar effective field theory (EFT) it is shown that the amplitude for a particle scattering off a two-body bound state, in leading order EFT, is entirely determined by two-body parameters. No three-body forces are required at the leading order. The arbitrary parameter present in the solution of the equation of Skorniakov and Ter-Martirosian for doublet channel nucleon-deuteron scattering is uniquely fixed by the EFT approach without involving any additional three-body data.
\end{abstract}

03.65.Nk, 11.10.Gh, 12.39.Fe, 13.75.Cs. 
Applications of effective field theory (EFT) to problems of nuclear physics have been under intensive investigations during the last few years. A review of recent developments (and references to the relevant papers) can be found in [四.

Generalisation of the EFT program to the three-body problem is not straightforward. In bosonic systems and in some fermionic channels (in particular the $J=1 / 2$ channel nucleondeuteron scattering) one encounters a non-trivial problem. While each leading order three-body diagram with re-summed two-body interactions is individually finite, the whole amplitude shows sensitivity to the ultraviolet cutoff. In [2] it was argued that the addition of an one-parameter three-body force counter-term at leading order is necessary and sufficient to eliminate this cut-off dependence.

The present paper considers the simple case of a non-relativistic scalar particle scattering off a two-body bound state and provides a solution of the above mentioned problem of sensitivity to the ultraviolet cut-off without introducing three-body forces into the leading order Lagrangian. Generalisation for $J=1 / 2$ channel nucleon-deuteron scattering is straightforward.

The discussion below borrows heavily from Ref. [3].

The Lagrangian of the considered EFT of non-relativistic self-interacting boson $\phi$ is given by the following expression [3]:

$$
\mathcal{L}=\phi^{\dagger}\left(i \partial_{0}+\frac{\vec{\nabla}^{2}}{2 M}\right) \phi-\frac{C_{0}}{2}\left(\phi^{\dagger} \phi\right)^{2}-\frac{D_{0}}{6}\left(\phi^{\dagger} \phi\right)^{3}+\ldots,
$$

where the ellipsis stands for terms with more derivatives and/or fields. Terms with more derivatives are suppressed at low momentum and terms with more fields do not contribute to the three-body amplitude. For the sake of convenience [4] one can rewrite this theory introducing a dummy field $T$ with the quantum numbers of two bosons (referred to as "dimeron" [3]),

$$
\begin{aligned}
\mathcal{L} & =\phi^{\dagger}\left(i \partial_{0}+\frac{\vec{\nabla}^{2}}{2 M}\right) \phi+\Delta T^{\dagger} T-\frac{g}{\sqrt{2}}\left(T^{\dagger} \phi \phi+\text { h.c. }\right) \\
& +h T^{\dagger} T \phi^{\dagger} \phi+\ldots
\end{aligned}
$$

The scale parameter $\Delta$ is included to give the field $T$ the usual mass dimension of a heavy field. Observables depend on the parameters of Eq. (2) only through the combinations $C_{0} \equiv g^{2} / \Delta=$ $4 \pi a_{2} / M$ and $D_{0} \equiv-3 h g^{2} / \Delta^{2}$.

The (bare) dimeron propagator is a constant $i / \Delta$ and the particle propagator is given by the usual non-relativistic expression $i /\left(p^{0}-p^{2} / 2 M\right)$. The dressing of the dimeron propagator is given in FIG.1 (a). Summing loop-diagrams, subtracting divergent integral at $p^{0}=\vec{p}^{2}=0$ and removing the cut-off one gets the following dressed dimeron propagator:

$$
i S(p)=\frac{1}{-\Delta^{R}+\frac{M g^{2}}{4 \pi} \sqrt{-M p^{0}+\frac{\vec{p}^{2}}{4}-i \epsilon}+i \epsilon} .
$$

Where $\Delta^{R}$ is the renormalised parameter ( $\Delta$ has absorbed the linear divergence). Attaching four boson lines to this dressed dimeron propagator one gets the two-particle scattering amplitude at leading order. This amplitude is identical to the effective range expansion truncated at the 


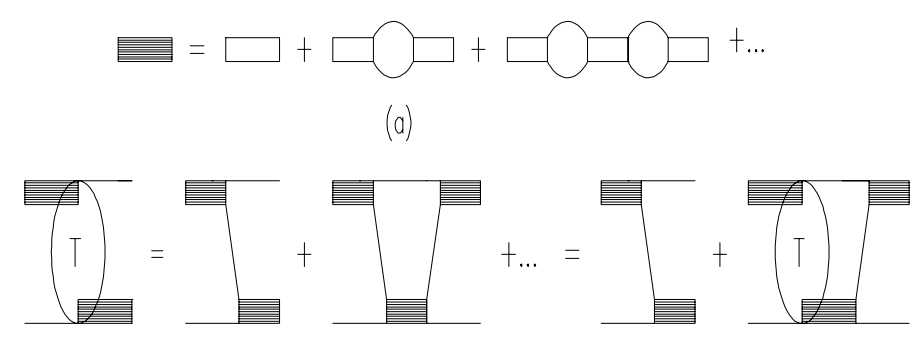

(b)

FIG. 1. (a) Dressing of the dimeron. (b) Diagrams contributing to the particle - bound-state scattering.

order of the scattering length. Further corrections give the next terms in the effective range expansion [5].

Standard power counting shows that diagrams which contribute to leading order calculations of particle - two-body bound state scattering are those illustrated in FIG.11 (b). The sum of all these diagrams satisfies the equation represented by the second equality in FIG.11(b) [6], [7 9]:

$$
a(p, k)=K(p, k)+\frac{2 \lambda}{\pi} \int_{0}^{\infty} d q K(p, q) \frac{q^{2}}{q^{2}-k^{2}-i \epsilon} a(q, k)
$$

where $k(p)$ is the incoming (outgoing) momentum, $M E=3 k^{2} / 4-1 / a_{2}^{2}$ is the total energy, $a(p=k, k)$ is the scattering amplitude, $a_{2}$ is the two-particle scattering length, and

$$
K(p, q)=\frac{4}{3}\left(\frac{1}{a_{2}}+\sqrt{\frac{3}{4} p^{2}-M E}\right) \frac{1}{p q} \ln \left(\frac{q^{2}+p q+p^{2}-M E}{q^{2}-q p+p^{2}-M E}\right) \equiv \frac{8}{3}\left(\frac{1}{a_{2}}+\gamma_{k}(p)\right) L_{E}(p, q)
$$

where the second equality introduces short notations for long expressions. Eq. (4) was first derived by Skorniakov and Ter-Martirosian [6] and has $\lambda=1$ for the boson case. Three nucleons in the spin $J=1 / 2$ channel obey a pair of integral equations with similar properties to this bosonic equation, and the spin $J=3 / 2$ channel corresponds to $\lambda=-1 / 2$.

It was shown in 10 that for $\lambda=1$ the homogeneous equation corresponding to Eq. (4) has a solution for arbitrary $E$. This solution is well-defined except for a normalisation constant and hence the solution of Eq. (4) contains an arbitrary parameter. So the sum of the diagrams in FIG.1 (b) is only one of the solutions and given the general solution of the Eq. (4), to find this sum one would have to fix the value of the arbitrary parameter appropriately.

The fact that the homogeneous equation corresponding to Eq. (4) has a solution for arbitrary $E$ is not surprising: since Eq. (4) corresponds to a coordinate space $\delta$-function potential, the use of the Thomas theorem [11] combined with the Efimov effect [12 explains the existence of solutions for arbitrary $E$. Note that two-body forces are not actually of zero range in EFT. Although Eq. (4) can be derived from the leading order Lagrangian of EFT, this equation is not a leading order approximation of a more general equation: there are no consistent equations for renormalised amplitudes in EFT if the cut-off is removed after renormalization. The problem is that EFT is a non-renormalizable theory in the traditional sense and hence to remove all divergences which occur in the equations for amplitudes one would need to include contributions of an infinite number of counter-terms at any finite order (except leading order) approximation. Hence EFT with removed cut-off describes the particle- two-body bound state scattering 
amplitude as a sum of an infinite number of diagrams. The EFT approach is concerned with Eq. (4) only because one of its solutions corresponds to this sum of diagrams.

Eq. (4) should be dealt with carefully, it actually has some features which are not characteristics of the full EFT.

Cut-off theory has a great advantage in that one can write down consistent equations, and the solutions of these equations are equivalent to the renormalised (with removed cut-off) amplitudes up to the order one is working with. If working with equations of cut-off theory it is necessary to keep the cut-off finite even though at leading order the cut-off can be removed, giving Eq. (四). As the equations with finite cut-off do not correspond to any system with local ( $\delta$-function type) potential, there are no three-body bound states with arbitrarily large negative energies. It should be clear from the above discussion that the solution of the homogeneous equation corresponding to equation (4), which exists for any value of the energy, does not carry any physical information. The existence of this solution is a result of the incorrect procedure of removing the cut-off in the leading order equations of the cut-off theory. The correct procedure would be to remove the cut-off in the equations of the cut-off theory (and hence switch to the old-fashioned approach to the renormalization procedure), and then consider the leading order; however as was mentioned above, there are no consistent equations if the cut-off is removed. Note that the amplitude determined from the equation of cut-off theory can contain some nonperturbative contributions in addition to the sum of the infinite number of diagrams drawn in FIG.1 (b) but, as should be clear from above discussion, these non-perturbative effects can not have anything to do with non-physical solutions of the homogeneous equation.

One can still use Eq. (4) to find the amplitude for particle - two-body bound state scattering, but one should keep in mind that it contains non-physical information encoded in the solution of the corresponding homogeneous equation.

As will be seen below the EFT approach fixes uniquely the arbitrary parameter present in the general solution of Eq. (4). This particular solution with an appropriately fixed value of the arbitrary parameter is the scattering amplitude.

One can study the asymptotic behaviour of $a(p, k)$ for large $p$. Up to terms decreasing as $p^{-1}$ the function $a(p, k)$ has the form [10]:

$$
a(p, k) \sim \sum_{i} A_{i}(k) p^{s_{i}}
$$

where $s_{i}$ are roots of the following equation:

$$
1-\frac{8 \lambda}{\sqrt{3}} \frac{\sin \frac{\pi s}{6}}{s \cos \frac{\pi s}{2}}=0 .
$$

The summation in Eq. (6) goes over all solutions of Eq. (7) for which $|\operatorname{Re} s|<1$.

Eq. (7) has two roots for which $|\operatorname{Res}|<1$ :

$$
s= \pm i s_{0}, \quad s_{0} \approx 1
$$

So, eq.(6) becomes:

$$
a(p, k) \sim A_{1}(k) p^{i s_{0}}+A_{2}(k) p^{-i s_{0}}
$$


One of the arbitrary constants $A_{1}(k)$ and $A_{2}(k)$ is determined by the other when this solution is joined to the solution in the region of small $p$. Hence the solution of Eq. (4) depends on a single arbitrary parameter. The asymptotic behaviour of the solution of the homogeneous equation corresponding to Eq. (4) is evidently the same.

Iterating the equation (4) one gets a series which is equivalent to the sum of the diagrams in FIG.11(b). As $s_{0}$ does not have an expansion in $\lambda$ with non-vanishing coefficients, it should be clear that for the sum of the considered diagrams (if it exists) the parameters $A_{1}(k)$ and $A_{2}(k)$ must be vanishing.

Hence the EFT with removed cut-off supports the conclusion drawn from general considerations, namely that the non-physical solution of the homogeneous equation has to be eliminated.

To find the sum of the considered infinite number of diagrams it is actually enough to have a particular solution of the inhomogeneous equation $a_{i h}$ and a solution of the homogeneous equation $a_{h}$. Remembering that up to a constant factor these two solutions have the same asymptotic behaviour one can construct the physical amplitude $a_{p h}$ by taking $a_{p h}=a_{i h}+B a_{h}$ and choosing the constant $B$ appropriately to reproduce a solution with the required asymptotic behaviour with vanishing $A_{1}(k)$ and $A_{2}(k)$. Note that there is only one solution with such asymptotic behaviour.

As was shown by G.S.Danilov [10] one can find $a(p, k)$ with a fixed value of the arbitrary parameter. This particular solution of the equation of Skorniakov and Ter-Martirosian for the energy $E$ is given by

$$
a(p, k)=\omega(p, k)+C(E) a_{E_{0}}(p)
$$

where $a_{E_{0}}(p)$ is a solution of the homogeneous equation for some energy $E_{0}$. The function $\omega(p, k)$ has the property

$$
p \omega(p, k) \rightarrow 0, \text { for } p \rightarrow \infty
$$

and satisfies the equation

$$
\frac{3}{8} \frac{\omega(p, k)}{\gamma_{k}(p)+1 / a_{2}}=\phi_{E}(p)+\frac{2}{\pi} \int_{0}^{\infty} \frac{\left(L_{E}(p, q)-L_{E}(p, k)\right) q^{2}}{q^{2}-k^{2}-i \epsilon} \omega(q, k) d q
$$

where

$$
\begin{gathered}
\phi_{E}(p)=\beta(k) L_{E}(p, k)-\frac{3}{8} \frac{C(E) a_{E_{0}}(p)}{\gamma_{k}(p)+1 / a_{2}}+L_{E}(p, k)+\frac{2}{\pi} \int_{0}^{\infty} \frac{L_{E}(p, q) q^{2}}{q^{2}-k^{2}-i \epsilon} a_{E_{0}}(q) d q \\
\beta(k)=-\frac{2 C(E)}{\pi} \int_{0}^{\infty} a_{E_{0}}(q)\left[\frac{k^{2}}{q^{2}-k^{2}-i \epsilon}-\frac{k_{0}^{2}}{q^{2}-k_{0}^{2}-i \epsilon}\right] d q \\
k_{0}^{2}=4\left(E_{0}+1 / a_{2}^{2}\right) / 3
\end{gathered}
$$

Eq. (11) can be used to determine $\omega(p, k)$ as it has an unique solution.

The equation for the determination of $C(E)$ has the form:

$$
C(E) \int_{0}^{\infty}\left[\frac{3}{8} \frac{a_{E_{0}}(p)}{\gamma_{k}(p)+1 / a_{2}}-\frac{2}{\pi} \int_{0}^{\infty} \frac{L_{E}(p, q) q^{2}}{q^{2}-k^{2}-i \epsilon} a_{E_{0}}(q) d q\right] \frac{a_{h}(p, k) p^{2}}{p^{2}-k^{2}-i \epsilon}=\frac{3 \pi}{16} \frac{a_{h}(k, k)}{2 / a_{2}}
$$


where $a_{h}(p, k)$ is a solution of the homogeneous equation for the energy $E$.

The next step is to take

$$
a_{p h}(p, k)=a(p, k)-B a_{h}(p, k)
$$

and adjust constant $B$ so that $a_{p h}(p, k)$ does not have oscillating asymptotic behaviour when $p \rightarrow \infty$. This solution $a_{p h}(p, k)$ corresponds to the sum of diagrams shown in FIG.1 (b). Note that $a_{p h}(p, k)$ does not depend on $E_{0}$. Eq. (四) has an unique solution with non-oscillating asymptotic behaviour. Choosing different value for $E_{0}$ one would get the same solution with non-oscillating asymptotic behaviour from Eq. (13) (as it is unique).

To solve Eq. (田) one could introduce a regularization. As this equation is singular (the corresponding homogeneous equation has a solution for an arbitrary value of the energy), nontrivial problems can be expected. The regularised equation has a unique solution, while the original equation has an infinite number of them. Which solution of un-regularised equation (if any) will one obtain when the regularization is removed from the solution of the regularised equation? There are an infinite number of different ways to regularize Eq. (田). It is not difficult to guess that (in general) different regularizations will lead to different solutions.

Following [3] one can introduce a particular regularization into Eq. (4):

$$
\begin{gathered}
a(p, k)=K(p, k)+\frac{2 \lambda}{\pi} \int_{0}^{\Lambda} d q K_{\Lambda}(p, q) \frac{q^{2}}{q^{2}-k^{2}-i \epsilon} a(q, k) \\
K_{\Lambda}(p, q)=\frac{8}{3}\left(\frac{1}{a_{2}}+\gamma_{k}(p)\right)\left(L_{E}(p, q)+\frac{H(\Lambda)}{\Lambda^{2}}\right)
\end{gathered}
$$

Here $\Lambda$ is a cut-off parameter and $H(\Lambda)$ is some function such that $\frac{H(\Lambda)}{\Lambda^{2}} \rightarrow 0$ when $\Lambda \rightarrow 0$. Note that while the $\frac{H(\Lambda)}{\Lambda^{2}}$ term was introduced as a three-body force in the Lagrangian in [3], Eq. (14) does not correspond to cut-off EFT, as in the latter case cut-off must be kept finite in the re-summed dimeron propagator as well.

To better understand some features of equations (44) and (14), it is useful to consider a simple matrix equation. Suppose "physical amplitude" is given by the following series:

$$
\left(\begin{array}{l}
a_{1} \\
a_{2}
\end{array}\right)=\left(\begin{array}{c}
\lambda \\
0
\end{array}\right)+\left(\begin{array}{c}
d \lambda \\
0
\end{array}\right)+\left(\begin{array}{c}
d^{2} \lambda \\
0
\end{array}\right)+\ldots=\left(\begin{array}{c}
\frac{\lambda}{1-d} \\
0
\end{array}\right)
$$

where $d \neq 1$. The "amplitude" given by Eq. (16) satisfies the following equation:

$$
\left(\begin{array}{l}
a_{1} \\
a_{2}
\end{array}\right)=\left(\begin{array}{l}
\lambda \\
0
\end{array}\right)+\left(\begin{array}{ll}
d & 0 \\
0 & 1
\end{array}\right)\left(\begin{array}{l}
a_{1} \\
a_{2}
\end{array}\right)
$$

The homogeneous equation

$$
\left(\begin{array}{l}
a_{1} \\
a_{2}
\end{array}\right)=\left(\begin{array}{ll}
d & 0 \\
0 & 1
\end{array}\right)\left(\begin{array}{l}
a_{1} \\
a_{2}
\end{array}\right)
$$

has a solution

$$
\left(\begin{array}{l}
0 \\
1
\end{array}\right)
$$


and the general solution of the inhomogeneous equation is

$$
a_{\text {gen }}=\left(\begin{array}{c}
\frac{\lambda}{1-d} \\
0
\end{array}\right)+C\left(\begin{array}{l}
0 \\
1
\end{array}\right)
$$

where $C$ is an arbitrary constant.

It is clear that Eq. (16) is just a particular solution with $C=0$.

The analogues of the diagrams are of course the terms of the series in Eq. (16). To find a sum of these "diagrams" one needs to find a particular solution with the bottom component equal to zero. If one finds any particular solution and a solution of the homogeneous equation, afterwards it is trivial to find a particular solution with the desired property: one just writes $a_{p h}=a_{\text {part }}-B a_{h}$ (where $a_{\text {part }}$ is a particular solution and $a_{h}$ is a solution of the homogeneous equation) and adjusts the arbitrary parameter $B$ in order to set bottom component of $a_{p h}$ equal to zero. This procedure is analogous to one suggested above for the integral equation (4).

On the other hand one can introduce a regularization into this simple equation:

$$
\left(\begin{array}{l}
a_{1} \\
a_{2}
\end{array}\right)=\left(\begin{array}{c}
\lambda \\
\epsilon f(\epsilon)
\end{array}\right)+\left(\begin{array}{ll}
d & 0 \\
0 & 1-\epsilon
\end{array}\right)\left(\begin{array}{l}
a_{1} \\
a_{2}
\end{array}\right) .
$$

Solving eq.(18) one obtains:

$$
\left(\begin{array}{c}
\frac{\lambda}{1-d} \\
f(\epsilon)
\end{array}\right)
$$

The limit $\epsilon \rightarrow 0$ leads to a particular solution. Choosing different $f$, one obtains different solutions: different regularizations lead to different solutions, but these different solutions just correspond to different values of the arbitrary parameter which is present in the general solution.

Taking $f(\epsilon)=C$ and changing the value of $C$ one would obtain any particular solution of the original un-regularised equation.

Now, one could take $f(\epsilon)=\cos (\ln \epsilon+\delta)$ where $\delta$ is arbitrary. $\epsilon f(\epsilon) \rightarrow 0$ when $\epsilon \rightarrow 0$, so if one takes the limit $\epsilon \rightarrow 0$ in equation (18) one gets the original un-regularised equation, but in the solution (19) the $\epsilon \rightarrow 0$ limit does not exist.

The limit $\epsilon \rightarrow 0$ does not exist in the solution for $f(\epsilon)=\cos (\ln \epsilon+\delta)$ because this choice of regularization is bad and the uncertainty occurring in this limit hardly has anything to do with the arbitrary parameter present in the general solution. Thus taking $f(\epsilon)=\cos (\ln \epsilon+\delta)$ one could come to the misleading conclusion that the original equation requires modification.

One could think that to find the "physical amplitude" the original "theory" has to be modified, that is, it is necessary to consider Eq. (18) with "three-body force" $f(\epsilon)=C$ and therefore an additional parameter $C$ is necessarily introduced. To fix this parameter would require one more "experimental input". As far as the "theory" describes the "experimental data" well, one would get $C=0$. Note that while the obtained solution for the "physical amplitude" would be correct, the conclusion about the additional parameter is of course incorrect. To find the "physical amplitude" one does not need to modify the original "theory". No additional "experimental input" is actually needed.

Switching back to Eq. (14), the authors of [3] found that if $H(\Lambda)=0$ then the solution of the cut-off equation does not converge as the cut-off parameter $\Lambda \rightarrow \infty$ (This is an analogue 
of $f(\epsilon)=\cos (\ln \epsilon+\delta)$ in the above toy model). Considering a non-trivial function $H(\Lambda)$ (the particular form of which is not important here), they obtained a solution which is well defined in the removed cut-off limit and which depends on an additional parameter (this is an analogue of $f(\epsilon)=C$ in the toy model). They concluded that renormalization has to be carried out more carefully. In particular they argued that to eliminate the mentioned cut-off dependence it is necessary to modify the leading order calculation by adding a three-body interaction term to the leading order Lagrangian. The free parameter introduced by the three-body interaction was fixed from additional experimental data. Analogously to the toy model, fitting an additional free parameter to the experimental data the value of this parameter which corresponds to the correct solution of the equation must be obtained, provided that leading order EFT calculations describe experimental data well. Hence the amplitude obtained in Ref. [3] should be reliable. On the other hand the conclusion about three body forces is not correct: one does not need to include three-body forces in leading order calculations. The additional "experimental input" is in fact predicted by EFT.

The problem of the cut-off sensitivity of the solution of Eq. (14) is hardly a concern of the given EFT approach, it is a general feature of singular equations of this kind. The correct procedure within the cut-off approach would be to retain a finite cut-off in all loop integrals, including those ones which were re-summed into the renormalised propagator of the dimeron field. The regularised equation (14) does not correspond to any cutoff EFT and hence fixing its problems by including terms into the Lagrangian of cutoff EFT is misleading. There is no reason to assume that different types of UV cutoff lead to different results for a given EFT.

If one is working in the framework of the old-fashioned renormalization scheme where the divergences are subtracted and then the cut-off is removed, then there are no equations for renormalised amplitudes, as EFT is a non-renormalizable field theory in the old-fashioned sense. Hence although one obtained an equation for the amplitude in the leading order, the solutions of this equation must be studied carefully to identify the physical part. On the other hand if one is working within the cut-off approach, one can formulate equations for the scattering amplitudes. It is very important to keep in mind that although in the leading order one can remove the cut-off, it is not a self-consistent procedure. If the cut-off is finite then there are no bound states with arbitrarily large negative energies. Thus the solutions of the homogeneous equations which exist for an arbitrary value of the energy, are the result of the in-consistent limiting procedure and have little to do with EFT.

To find the bound state energy one needs to find a pole of the scattering amplitude. While the homogeneous equation corresponding to Eq. (四) has a non-physical solution for any value of the energy, it should have additional physical solutions for the energies of physical bound states. Hence to find the bound state energy one needs to look for those values of energy for which the homogeneous equation has double solutions: two eigenvectors (one physical and one non-physical) corresponding to one eigenvalue.

As should be clear from the above discussions, the EFT (with removed cut-off) approach to spinless particle - two-body bound state scattering problem at leading order leads to the equation of Skorniakov and Ter-Martirosian together with a boundary condition at the origin (in configuration space) which eliminates the oscillating behaviour. Hence EFT resolves quite naturally the problem of the choice for the arbitrary parameter which is present in the general solution of this equation for any values of the energy. Note that in the original approach by Skorniakov and Ter-Martirosian the boundary condition has to be introduced by hand [10]. 
The homogeneous equation corresponding to the equation of Skorniakov and TerMartirosian has a solution for any value of the energy, but this solution does not correspond to the pole of the physical scattering amplitude obtained from EFT. As was observed in [11] a long time ago, there is no lower bound for energies of the solutions of the Schrödinger equation for three particles when the two-body potential is singular. As should be clear from the above discussion, these unbounded solutions are not physical at least from the point of view of effective field theory.

\section{ACKNOWLEDGEMENTS}

The author would like to thank D.Phillips for useful discussions and B.Blankleider and S.Gunner for comments on the manuscript.

This work was carried out whilst the author was a recipient of an Overseas Postgraduate Research Scholarship and a Flinders University Research Scholarship at the Flinders University of South Australia. 


\section{REFERENCES}

[1] U. van Kolck, nucl-th/9902015;

[2] P.F. Bedaque, H.-W. Hammer, and U. van Kolck, Phys.Rev.Lett. 82 (1999) 463, nucl-th/9809025;

[3] P.F. Bedaque, H.-W. Hammer, and U. van Kolck, nucl-th/9811046;

[4] D.B. Kaplan, Nucl. Phys. B494 (1997) 471;

[5] U. van Kolck, in Proceedings of the Workshop on Chiral Dynamics 1997, Theory and Experiment, A. Bernstein, D. Drechsel, and T. Walcher (editors), Springer-Verlag, 1998, hep-ph/9711222; in Ref. [13]; nucl-th/9808007;

[6] G.V. Skorniakov and K.A. Ter-Martirosian, Sov. Phys. JETP 4 (1957) 648;

[7] P.F. Bedaque, in Ref. [13], nucl-th/9806041;

[8] P.F. Bedaque and U. van Kolck, Phys. Lett. B428 (1998) 221;

[9] P.F. Bedaque, H.-W. Hammer, and U. van Kolck, Phys. Rev. C58 (1998) R641;

[10] G.S. Danilov, Sov. Phys. JETP 13 (1961) 349;

[11] L.H. Thomas, Phys. Rev. 47 (1935) 903;

[12] V.N. Efimov, Sov. J. Nucl. Phys. 12 (1971) 589;

[13] "Nuclear Physics with Effective Field Theory", R. Seki, U. van Kolck, and M.J. Savage (editors), World Scientific, 1998. 